\title{
The multidisciplinary management of pre-sacral lesions, including percutaneous biopsy
}

\author{
A. N. Murphy, A. Sterling, E. Kavanagh, H. Fenlon, C. Cronin. \\ Mater Misericordiae University hospital, Phibsborough, Dublin, Ireland
}

Background

- Pre-sacral lesions may be congenital or acquired, benign or malignant, and can originate from osseous, neurogenic or soft tissue structures.

- A multidisciplinary team (MDT) management approach is key, with input from radiologists, surgical specialists, pathologists, and Medical and Radiation Oncologists.

Preoperative biopsy; To biopsy or not to biopsy?

- In the past pre-operative biopsy was considered contraindicated due to the theoretical and anecdotal risk of seeding which restricts surgical options ${ }^{4,9}$

- Predominantly cystic lesions should not be biopsied due to the risk of complication such as secondary infection, fistula formation or meningitis and unlikely benefit as the vast majority are benign.

- However if imaging features are suggestive of a solid malignant lesion such as Ewing sarcoma, osteosarcoma, or neurofibrosarcoma, pre-operative biopsy should be considered as these conditions may benefit from neo-adjuvant chemotherapy ${ }^{1,3}$.
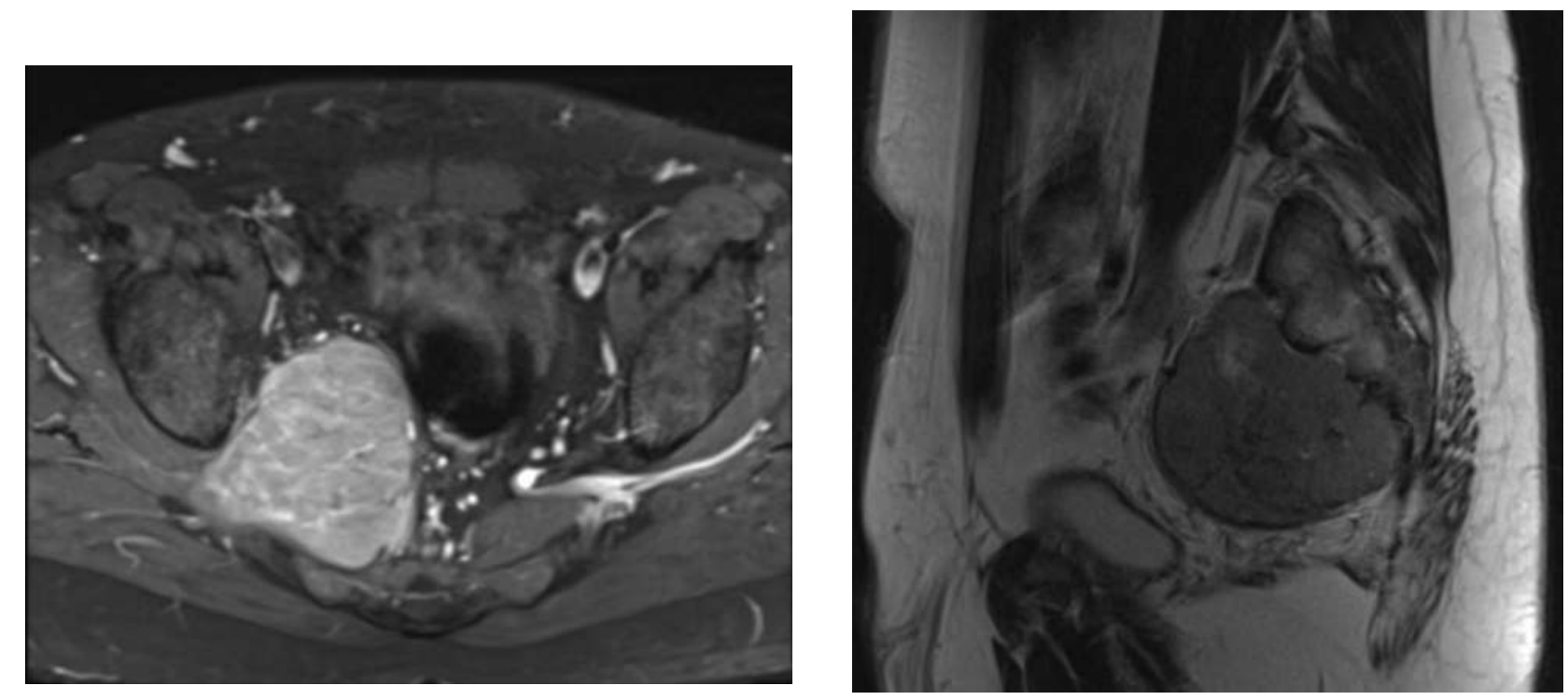

This 33 year old male presented with sensory loss over the right S1 nerve distribution. MRI shows an aggressive appearing heterogenous soft tissue mass in the right pre-sacral region involving the right piriformis, closely related to the right S1 neural exit foramen. This was biopsied and diagnosed as an Ewing's tumour which allowed appropriate management and resection.

- There is emerging evidence that chordomas and chondrosarcomas, previously thought to be chemoresistant, show promising results with tyrosine kinase inhibitor neoadjuvant therapy ${ }^{1,4}$.

- If surgical resection is not clinically appropriate, biopsy can guide palliate chemotherapy or radiotherapy.
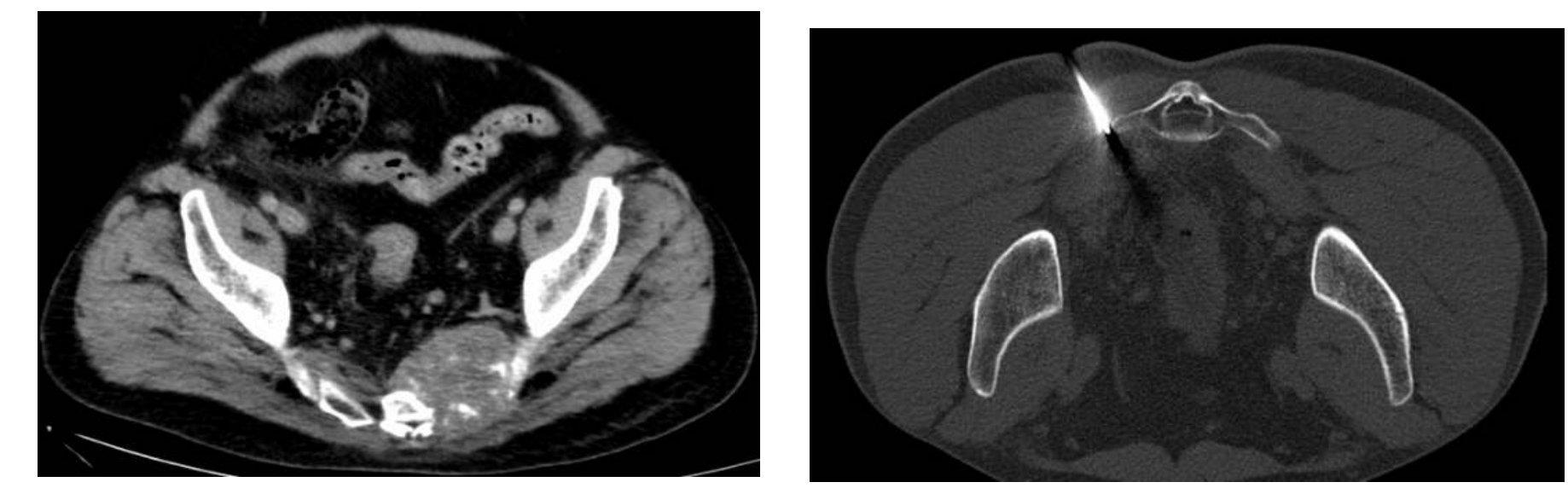

This $4 \mathrm{I}$ year old male had a recent history of rectal cancer treated with neoadjuvant chemoradiotherapy however had had a poor response. CT displays a partially calcified deposit in the left pre-sacral region, eroding into bone. This was biopsied and metastatic disease confirmed.
Surgical management of pre-sacral lesions.

- Total resection is the treatment of choice for most pre-sacral lesions as even if the lesion is benign it can cause complications such as infection, fistulation, mass effect and undergo malignant transformation $1,3,5$.

- Colorectal surgeons generally manage pre-sacral lesions, however depending on the exact origin and pelvic organ involvement, input may be needed from orthopaedic, vascular, urology or gynaecology specialists ${ }^{1,3}$.

\section{Surgical approach.}

- The relationship of the lesion to the third sacral segment (S3) is important for surgical planning; lesions at or above $\mathrm{S} 3$ call for an anterior approach, while lesions below call for a posterior approach. When there is disease above and below S3, a combined approach is called for ${ }^{1,4}$.

Post surgical complications.

- There is a high post-operative morbidity. For anatomic reasons, postoperative impairment of bladder, bowel, sexual, and ambulatory function is inevitable.

- Although unilateral resection of all the sacral nerve roots will not compromise fecal and urinary function, if both S-3 nerve roots are resected, the external anal sphincter will not function and the patient will become incontinent.

Radiology report details addressing pre-sacral lesions.

- Tissue of origin: Nervous tissue, bone or soft tissue?

- Characteristics: Solid/cystic, fat/haemorrhage/calcification, enhancement pattern?

- Benign or aggressive appearance?

- Surgical considerations:

- Site - in relation to S3 Vertebral Body

- Extent - in relation to S3 and other sacral levels.

- Size - 3 dimensions

- Detail of extent of invasion into:

- Sacrum, coccyx and lumbar spine.

- Nerve roots and spinal canal.

- Pelvic sidewall - piriformis, obturator internus muscles, sacrotuberous and sacrospinous ligaments.

- Local viscera - rectum, uterus, ureters, internal iliac vessels.

- Possible ureteric involvement and potential need for ureteric stents to aid in the intraoperative identification of the ureters.

- Venous obstruction / Deep Venous Thrombosis.

Conclusions.

An MDT approach to the management of pre-sacral lesions is key. Advances in imaging and percutaneous biopsy techniques have improved the evaluation and management of pre-sacral lesions in the recent past. Treatment is surgical; due to their complexity, many presacral lesions need multiple sub-specialists' input. Finally, the relationship of the lesion to $\mathrm{S} 3$ is imperative. 